\title{
Proof of a decomposition theorem for symmetric tensors on spaces with constant curvature
}

\author{
Norbert Straumann \\ Institute for Theoretical Physics University of Zurich, \\ Winterthurerstrasse 190, CH-8057 Zurich, Switzerland
}

May 28, 2018

\begin{abstract}
In cosmological perturbation theory a first major step consists in the decomposition of the various perturbation amplitudes into scalar, vector and tensor perturbations, which mutually decouple. In performing this decomposition one uses - beside the Hodge decomposition for one-forms - an analogous decomposition of symmetric tensor fields of second rank on Riemannian manifolds with constant curvature. While the uniqueness of such a decomposition follows from Gauss' theorem, a rigorous existence proof is not obvious. In this note we establish this for smooth tensor fields, by making use of some important results for linear elliptic differential equations.
\end{abstract}

\section{The decomposition theorem}

In cosmological perturbation theory one can regard the various perturbation amplitudes as time dependent tensor fields on a three-dimensional Riemannian space $(M, g)$ of constant curvature $K$ (see, e.g., [1]). For skew-symmetric tensor fields (p-forms) there is on arbitrary compact Riemannian manifolds the profound Hodge decomposition into an orthogonal direct sum of exact, coexact, and harmonic forms. No analogous decomposition for symmetric tensor fields, say, is available in general. However, when the space has constant curvature, a symmetric tensor field $t_{i j}$ can be decomposed as follows:

$$
t_{i j}=t_{i j}^{(S)}+t_{i j}^{(V)}+t_{i j}^{(T)}
$$


where

$$
\begin{aligned}
t_{i j}^{(S)} & =\frac{1}{3} t^{k}{ }_{k} g_{i j}+\left(\nabla_{i} \nabla_{j}-\frac{1}{3} g_{i j} \nabla^{2}\right) f, \\
t_{i j}^{(V)} & =\nabla_{i} \xi_{j}+\nabla_{j} \xi_{i}, \\
t_{i j}^{(T)} & : \quad t^{(T) i}{ }_{i}=0 ; \quad \nabla_{j} t^{(T) i j}=0 .
\end{aligned}
$$

In these equations $f$ is a function on $M$ and $\xi^{i}$ a vector field with vanishing divergence; $\nabla^{2}$ denotes $g^{i j} \nabla_{i} \nabla_{j}$ on $(M, g)$. (Note that this does not agree with the Laplace-Beltrami operator $\triangle$ for differential forms, except on functions. The difference is given by the Weitzenböck formula [2]. For general tensor fields $\nabla^{2}$ is the natural extension of the Laplace operator on functions.) The three components are easily shown to be orthogonal to each other with respect to the scalar product.

$$
\langle t, s\rangle=\int_{\Sigma} t_{i j} s^{i j} d \mu
$$

where $\mu$ is the Riemannian measure for the metric $g$. This fact implies that the decomposition of $t_{i j}$ is unique. Below we give a rigorous existence proof.

\subsection{Some tools}

In this subsection $(M, g)$ can be an arbitrary compact (closed) Riemannian manifold. On this we consider operators

$$
L=-\triangle+k, \quad k \in \mathbb{R} .
$$

Specializing existence and regularity results from the theory of elliptic partial differentials equations, established for instance in chapter 5 of [3], the following holds:

(i) The equation $L u=f$, with $f \in C^{\infty}(M)$ has a solution $u \in C^{\infty}(M)$ if and only if $f$ is orthogonal to the smooth functions $v$ satisfying $L v=0$. In particular, $\triangle\left(C^{\infty}(M)\right)=H^{\perp}$ : the orthogonal complement of the harmonic functions $H$ in $C^{\infty}(M)$.

(ii) In the space of smooth functions the equation $L u=f$ has always a unique solution, if $k$ is not an eigenvalue of the operator $\triangle$.

(iii) If $k$ is an eigenvalue of $\triangle$, and $f$ is orthogonal to the smooth eigenfunctions $w$ of $\triangle$ with eigenvalue $k$, then there are smooth solutions of $L u=f$. Any two of them differ by such an eigenfunction $w$.

In passing we note that $L^{2}$-completeness, as well as uniform completeness of the smooth eigenfunctions of $\triangle$ holds. We will, however, not use this fact. We also recall that harmonic functions on $M$ are constant. 


\subsection{Proof of the decomposition theorem}

Let now $(M, g)$ be a Riemannian space of constant curvature $K$. Then the Ricci tensor and Ricci scalar are given by

$$
R_{i j}=(n-1) K g_{i j}, \quad R=n(n-1) K .
$$

Below we shall use the following consequence of the Ricci identity:

$$
\nabla^{2} \nabla_{i} \omega_{j}=\nabla_{i} \nabla^{2} \omega_{j}+K\left[(n-1) \nabla_{i} \omega_{j}+2 \nabla_{j} \omega_{i}-2 g_{i j} \nabla^{k} \omega_{k}\right] .
$$

For definiteness we consider the compact case of an n-dimensional sphere. (In the non-compact case one has to add fall-off conditions.)

The decomposition theorem follows immediately, once we have shown that for any symmetric traceless tensor $t_{i j}$ there exists a covariant vector field $A_{i}$, such that

$$
t_{i j}-\nabla_{i} A_{j}-\nabla_{j} A+\frac{2}{n} g_{i j} \nabla^{k} A_{k}
$$

is transversal, i.e., satisfies the second equation in (4). (Apply in a second step the decomposition (12) below.) With the help of the Ricci identity and (7) this condition can be written as

$$
\left[\nabla^{2}+(n-1) K\right] A_{i}+\left(1-\frac{2}{n}\right) \nabla_{i}\left(\nabla_{j} A^{j}\right)=\nabla^{j} t_{i j}
$$

So, the existence of a decomposition (11) is equivalent to the question of whether there is a covariant vector field satisfying equation (10). We now show that this question has a positive answer.

Applying $\nabla^{i}$ on (10), and using as a special case of (8) the identity $\nabla^{i} \nabla^{2} A_{i}=\nabla^{2} \nabla^{i} A_{i}+(n-1) K \nabla_{i} A^{i}$, we obtain

$$
(\triangle+n K) \nabla^{i} A_{i}=\frac{n}{2(n-1)} \nabla^{i} \nabla^{j} t_{i j} .
$$

As a special case of the Hodge decomposition, $A_{i}$ can be uniquely decomposed into an direct orthogonal sum of the form

$$
A_{i}=V_{i}+\nabla_{i} S, \quad \nabla^{i} V_{i}=0,
$$

whence

$$
\nabla^{i} A_{i}=\triangle S
$$

Then (11) becomes

$$
\triangle[(\triangle+n K) S]=\frac{n}{2(n-1)} \nabla^{i} \nabla^{j} t_{i j} .
$$


Note that $\lambda_{1}:=-n K$ is an eigenvalue of $\triangle$. Since the right-hand side of this equation is by Gauss' theorem in $H^{\perp}$, equation (14) has, up to an additive constant, a unique solutions for $(\triangle+n K) S$. Equation (10) can be rewritten as

$$
\left[\nabla^{2}+(n-1) K\right] V_{i}=\nabla^{j} t_{i j}-\frac{2(n-1)}{n} \nabla_{i}(\triangle S+n K S) .
$$

There are certainly solutions of (14) and (15). For the latter one has to use property (ii) of Sect. 1.1 for 1-forms. The left-hand side of (15) is equal to the operator $\triangle+2(n-1) K$ applied on the 1 -form belonging to $V_{i}$. For any solution of the two equations, $A_{i}$ given by (12) then satisfies equation (10). Indeed, applying $\nabla^{i}$ on (15) and using (14) leads to $[\triangle+2(n-1) K] \nabla^{i} V_{i}=0$, hence $\nabla^{i} V_{i}=0$. Then, the definition (12) implies $\nabla^{i} A_{i}=\triangle S$. If one now replaces $V_{i}$ in (15) by $V_{i}=A_{i}-\nabla_{i} S$ and sets $\triangle S=\nabla^{i} A_{i}$ in the resulting equation, one recovers (10).

This concludes the proof.

\section{References}

[1] N. Straumann, From Primordial Quantum Fluctuations to the Anisotropies of the Cosmic Microwave Background Radiation, Ann. Phys. (Leipzig) 15, 701-847 (2006).

[2] J. Jost, Riemannian Geometry and Geometric Analysis, 4th ed. (Springer, Berlin, 2005), Sect. 3.3.

[3] M.E. Taylor, Partial Differential Equations, Basic Theory, Texts in Applied Mathematics 23 (Springer, Berlin, 1996). 